\title{
Extreme current measurements using a fibre optics current sensor
}

\author{
F. Briffod, D. Alasia, L. Thévenaz, G. Cuénoud* and Ph. Robert \\ EPFL Swiss Federal Institute of Technology, Laboratory of Metrology and Photonics, \\ $\mathrm{CH}-1015$ Lausanne, Switzerland \\ * LEM High Current Systems, Ch. des Aulx 18, \\ $\mathrm{CH}-1228$ Plan-les-Ouates, Switzerland
}

Tel : [++41 21] 6937606 Fax : [++41 21] 6932614 E-mail : fabien.briffod@epfl.ch

\begin{abstract}
The aim of this paper is to demonstrate that an optical fibre current sensor can measure current over $500 \mathrm{kA}$ with an accuracy of $0.2 \%$; this was never achieved using a traditional non-optical sensor.
\end{abstract}

\section{INTRODUCTION}

This paper presents measurements of very high current using an interferometric Sagnac fibre optics current sensor developed by the Laboratory of Metrology and Photonics in the Swiss Federal Institute of Technology, in collaboration with Trench Switzerland AG. These measurements were performed at LEM High Current Systems, in Geneva, Switzerland and demonstrate that currents exceeding 500kA can be measured with an optical fibre sensor, out of the range reached by classical sensors.

The sensor presented here is designed to measure high DC and AC currents up to $\pm 500 \mathrm{kA}$. The objective of this sensor is to obtain a $\pm 0.2 \%$ accuracy over the full $\pm 500 \mathrm{kA}$ range, for a temperature range between 0 and 50 degC ( -20 to $80 \mathrm{degC}$ for the sensing fibre), to comply with the end user requirements.

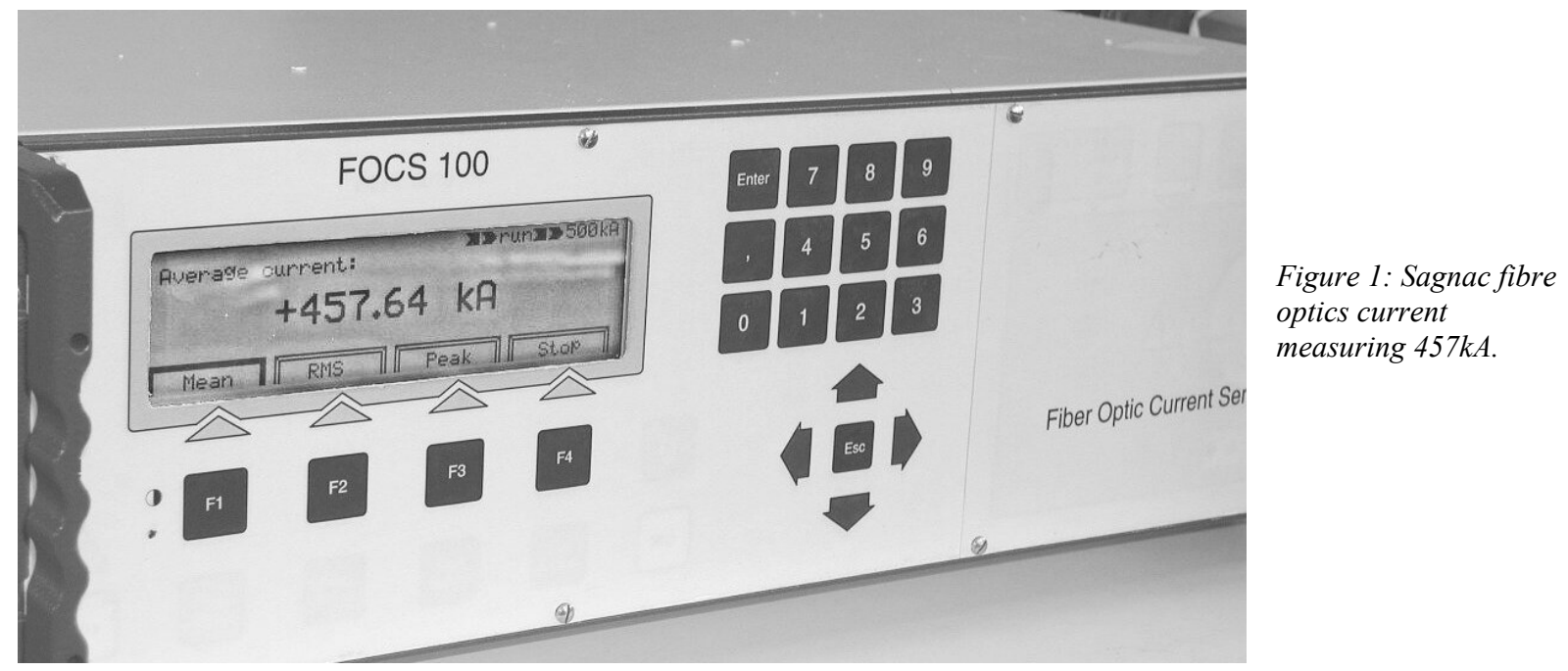

As a result of the developments on optical gyroscopes [1,2] it is now well-known that the Sagnac interferometer has the key advantage to be only sensitive to nonreciprocal effects, such as rotation and Faraday effect. As a result, this interferometer is intrinsically insensitive to reciprocal effects such as fibre elongation due to thermal expansion or mechanical solicitations.

The main issue that prevented the development of a reliable instrument so far is related to the fibre birefringence. It turns out that such a sensor can only give unbiased measurements, provided that the light polarisation is maintained circular all along the sensing fibre loop. Many solutions have been proposed to compensate the effect of birefringence, either optically or through a set of measurements and some calculations $[3,4,5]$. But these techniques are limited to homogeneous linear birefringence along the sensing fibre, so that they turn out to be widely inapplicable in actual conditions in which the fibre birefringence is basically random. 
Fortunately the detrimental effect of polarisation mode dispersion in telecommunication systems had led to a big effort for manufacturing very low birefringence fibres at low cost. The remaining birefringence of such fibres turns out to be still too large for the proper operation of a current sensor. But it can now be widely rendered negligible by annealing [6] or mechanically twisting the fibre [7], so that a circular or freely-rotating linear polarisation is maintained over the entire fibre length. The aim of this paper is to demonstrate that an optical fibre current sensor can measure current over 500kA; this was never achieved using a traditional non-optical sensor.

\section{SETUP}

The diagram of the realised fibre current sensor is shown in Figure 2. The configuration is very similar to that used in fibre optics gyroscopes, with the particularity to propagate the light through the sensing loop using a circular polarisation. This particularity requires a proper preparation of the light polarisation. For current sensing the fibre loop must enclose the probed electrical conductor. The circulation of the current magnetic field along the fibre causes a phase lag between circularly polarised waves propagating in opposite directions, as a result of the Faraday effect. After propagation into the sensor head, the two waves recombine, resulting in an interference signal to be processed.

In absence of any electrical current the interferometer is fully balanced and its sensitivity to a differential phase shift is zero. An electro-optic phase modulator is thus used to bias the response of the interferometer, so that a maximum sensitivity for small electrical current is obtained, technique similar to that used in fibre gyros [8]. A standard lock-in detection at $f_{m}$ and $2 f_{m}$ followed by a suitable signal processing provides a value for the electrical current independent on any change in the light source intensity. The electro-optic phase modulation turns out to be temperature-dependent to an unacceptable extent, so that the Gyro-type modulator has to be temperature-controlled. The electro-optic modulator also achieves the splitting-recombination functions of the interferometer and transmits the light only in a well defined linear polarisation state.

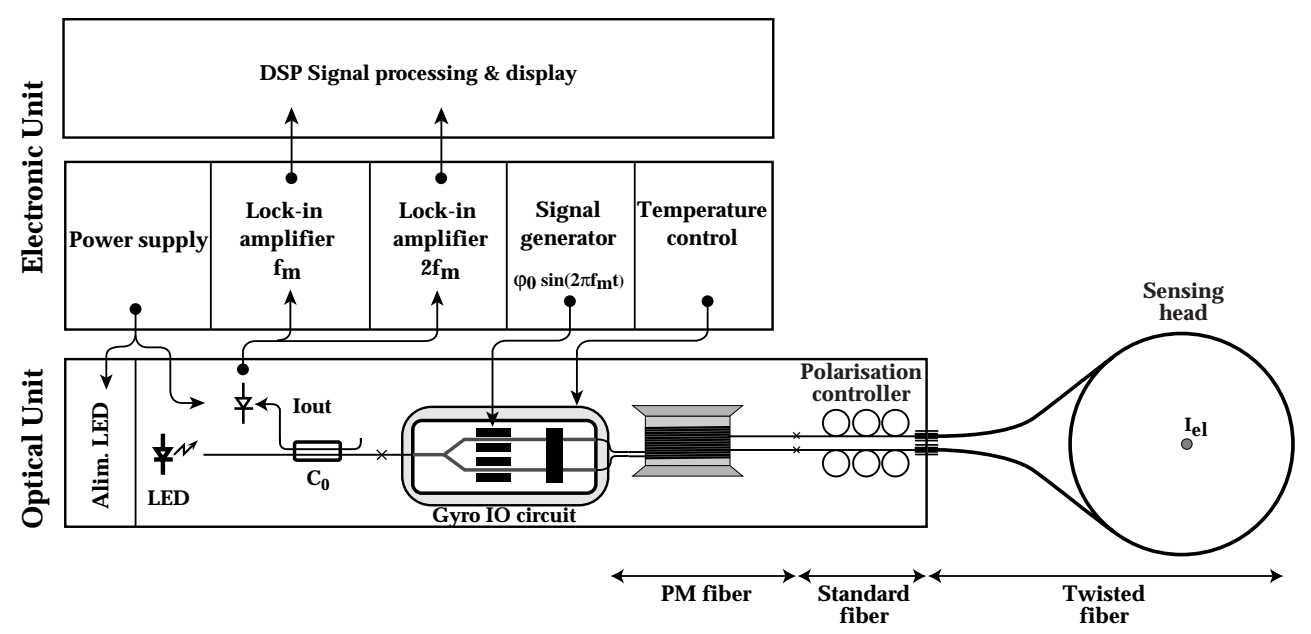

Figure 2: Block diagram of the Sagnac interferometric current sensor in the field measurement configuration.

Polarisation maintaining fibres (PM fibre) are used to transmit the linearly polarised light from the electro-optic phase modulator (GYRO circuit) to high stability polarisation controllers dedicated to the preparation of the proper polarisation for the sensing fibre. With respect to classical configurations some functional improvements were brought up. The sensing fibre is a simple cable containing a mechanically twisted fibre and terminated with connectors. Hence, on-site installation is easily performed by wrapping the fibre around the conductor and does not require the interruption of the probed electrical circuit. To grant a circular polarisation in the sensing head, polarisation controllers are carefully set, so that they transform the linear polarisation into a circular polarisation within the sensing fibre, by moreover compensating any spurious birefringence induced by the connectors.

\section{MEASUREMENTS}

The experimental configuration used for this measurement is represented in figure 3 . In this configuration, the magnetic field is in no way homogeneous along the fibre. Two different coil types are used, placed at different positions along the fibre, demonstrating that this sensor exactly measures the integral of the magnetic field along the fibre. Two coils of 5'000 turns are used with a current up to $2.5 \mathrm{~A}$ through each, resulting in an equivalent 
current of $25 \mathrm{kA}$. Besides, 8 other coils of 250 turns are used (4 coils are mounted in series and the two groups of 4 coils are mounted in parallel). A current up to $80 \mathrm{~A}$ is used through these coils, resulting in an equivalent current of $80 \mathrm{kA}$. Finally, 5 turns of sensing fibre enclose these coils, resulting in an equivalent current up to $525 \mathrm{kA}$.

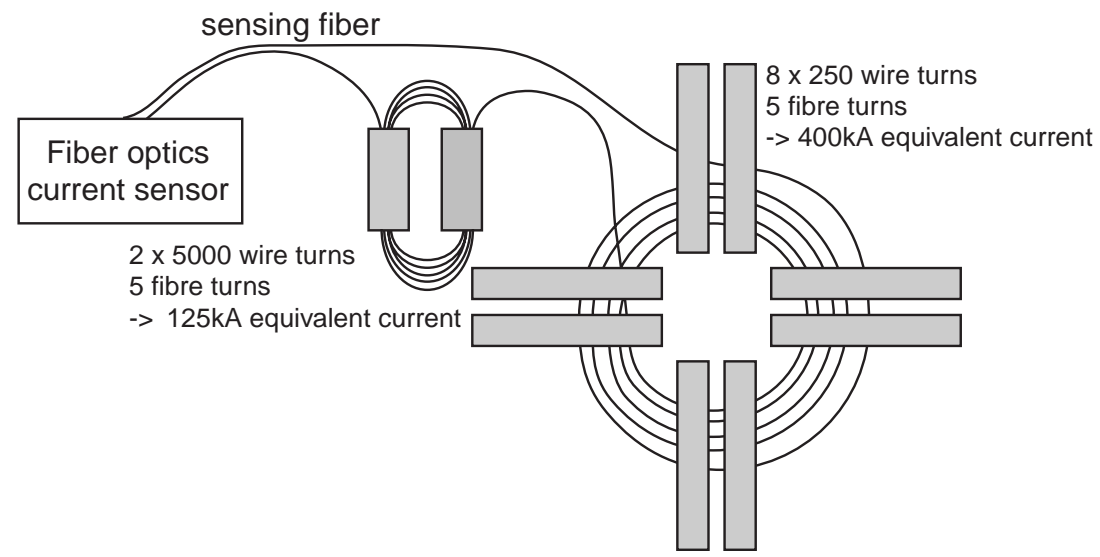

Figure 3: Experimental configuration for the measurement up to $500 \mathrm{kA}$.

Figure 4 shows the response of the sensor up to more than 500kA, obtained for DC current, demonstrating the excellent linearity of the instrument and its high dynamics range.

Figure 5 shows the difference between the reference current and the optically measured current. The manner the two currents are measured explains the remaining scattering of points: the reference current is not stabilized and the reference and optical measurements of the current are not simultaneous. Thus we had to collect measurements manually while the current was likely to fluctuate. In spite of these experimental conditions, we obtained a linearity better than $0.1 \%$.

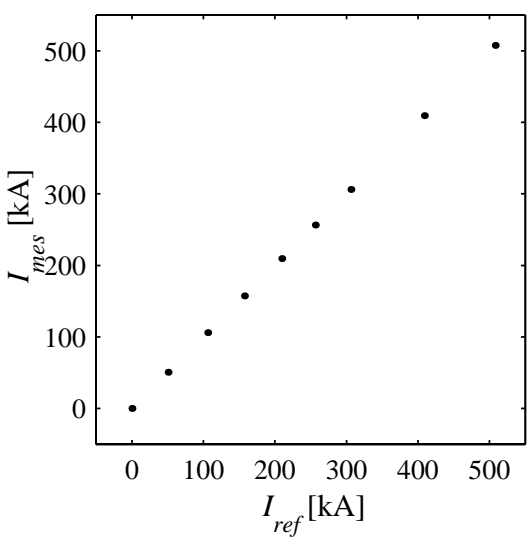

Figure 4: Response of the sensor to DC current up to $500 \mathrm{kA}$. $I_{\text {ref }}$ is the reference electrical current and $I_{m e s}$ is the optically measured current.

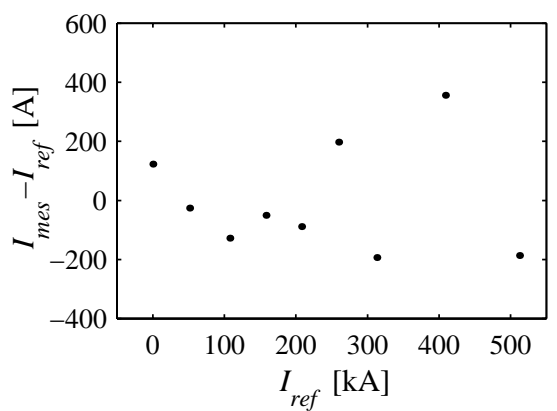

Figure 5: Non-linearity of a DC current measured up to $500 \mathrm{kA}$.

Thermal and mechanical stability and reproducibility are key-issues for fibre current sensors and a full set of measurement was carried out to emphasise problems related to these issues.

Figure 6 shows the fluctuations of the measured value on a long-term basis, at constant ambient temperature, resulting on a low drift of approximately $0.2 \%$

Another critical test is shown in Figure 7, in which the sensing fibre was subject to strong temperature change. In this experiment, we performed a temperature cycle from ambient temperature, rising up to 50 degC, falling down to $5 \mathrm{degC}$ and finally reaching ambient temperature again. We observe a drift of $0.2 \%$ approximately.

The observed hysteresis is partially due to the offset in the temperature measurement that is not exactly that of the sensing fiber. We had to wait some delay until the temperature was enough stabilized for a better measurement. 


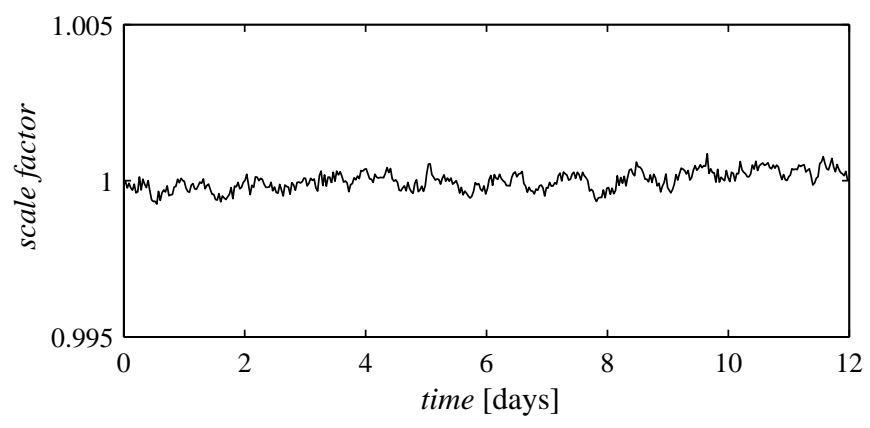

Figure 6: Variation of the scale factor on 12 days measurement.

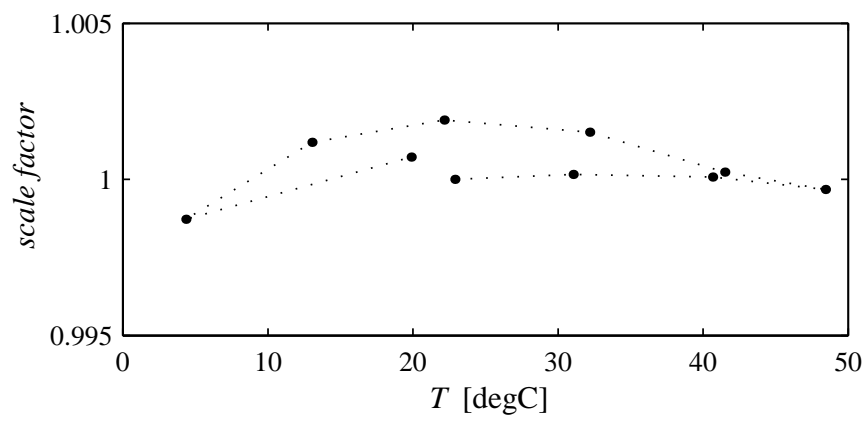

Figure 7: Variation of the scale factor due to temperature

change of the sensing fiber.

\section{CONCLUSION}

This paper shows the capability and performances of a Sagnac interferometric current sensor for very high current up to 500kA. The handling of the flexible sensing fibre is easy and fulfils the requirement of the end user. Results show that an accuracy of $0.2 \%$ over an extended temperature change is now obtained. But most of all, these measurements demonstrate that a new solution exist to measure or calibrate industrial installations where the measurement of a DC current higher than 100kA is required, range in which non-optical sensors fail. This solution, compared to standard ones, shows the further advantage to offer a very light sensor, easy and quick to install, together with an excellent accuracy.

\section{REFERENCES}

[1] H. J. Arditty and H. C. Lefèvre, "Sagnac effect in fibre gyroscopes", Optics Letters, vol. 6, pp. 401-403, 1981

[2] W. R. Leeb, G. Schiffner and E. Scheiterer, "Optical Fibre gyroscopes: Sagnac or Fizeau effect", Applied Optics, vol. 18, pp. 1293-1295, 1979

[3] P.-A. Nicati, Ph. Robert, "Stabilized current sensor using Sagnac interferometer", Journal of Physics, vol. 21, n. 8; pp. 791-796, August 1988

[4] Z. B. Ren and Ph. Robert, "Polarization multiplexing applied to a fiber current sensor", Optics Letters, vol. 14 , n. 21, pp. 1228-1230, 1989

[5] P.A. Nicati and Ph. Robert, "Stabilized Sagnac optical fibre current sensor using one phase and two amplitude modulations", $8^{\text {th }}$ OFS Proceedings, pp. 402-405, Monterey, 1992

[6] D. Tang, A. H. Rose, G. W. Day, S. M. Etzel, "Annealing of Linear Birefringence in Single-Mode Fibre Coils: Application to Optical Fibre Current Sensor", Journal of Lightwave Technology, vol. 9, n. 8, pp. 1031-1037, August 1991

[7] R. Urlich, A. Simon, "Polarization optics of twisted single-mode fibres", Applied Optics, vol. 18, n. 13, pp. 2241-2251, July 1, 1979

[8] R. Ulrich, "Fiber-optic rotation sensing with low drift", Optics Letters, vol. 5(5), pp. 173-175, 1980 